\title{
A design approach for integrated CMOS LC-tank oscillators using bifurcation analysis
}

\author{
M. Prochaska, K. Bohle, and W. Mathis \\ University of Hannover, Institute of Theoretical Electrical Engineering, Appelstraße 9A, 30167 Hannover, Germany
}

\begin{abstract}
Electrical oscillators play a decisive role in integrated transceivers for wired and wireless communication systems. In this context the study of fully integrated differential VCOs has received attention. In this paper formulas for investigations of the stability as well as the amplitude of CMOS LC tank oscillators are derived, where an overall model of nonlinear gain elements is used. By means of these results we are able to present an improved design approach which gives a deeper insight into the functionality of LC tank VCOs.
\end{abstract}

\section{Introduction}

The demand for ever-higher frequencies, higher levels of integration, low power consumption and low costs poses a challenge for the design and implementation of RF oscillators. To fulfill these requirements - especially in the field of mobile communication - in recent years fully integrated cross coupled voltage controlled LC tank oscillators received attention as shown in Razavi (1997) and van der Tang et al. (2003). Predominantly piecewise linear models are used which are based on heuristic methods. For the calculation of the amplitude the method of describing function is common practice, where typically higher-order harmonics are discarded. In order to guarantee a steady state oscillation the Barkhausen criterion is well-established which is also based on a linearized model of the oscillatory circuit, see e.g. Tietze and Schenk (2004). Since the nonlinearity is essential for the operation of electrical oscillators (Mathis and Russer, 2005), we present an alternative approach which uses a nonlinear overall model of the active elements comparable with Buonomo and LoSchiavo (2002) and Buonomo and LoSchiavo (2003). Starting from a nonlinear model of the transistors the VCO is modeled by a nonlinear dynamical system and a

Correspondence to: M. Prochaska

(prochaska@tet.uni-hannover.de) stability condition as well as a formula for the tank amplitude is derived. For this purpose symbolic algorithms are used, which are implemented by computer algebra. Compared to Prochaska et al. (2005) we present an enhanced methodology for CMOS technology. In order to demonstrate the practicability of our approach we have implemented our technique into a well-known design concept introduced in Hajimiri and Lee (1999) and Ham and Hajimiri (2001). It turns out that by means of our results common design schemes can be improved, since the presented methodology provides a deeper insight into the behavior of fully integrated LC tank oscillators.

\section{Nonlinear analysis}

There are lots of implementations of CMOS differential LC tank oscillators which differ wildly with respect to their resonance frequency, noise behavior or power consumption. Without a loss of generality we have decided to analyze a VCO introduced in de Muer et al. (1998) (Fig. 1), since for the verification of our results a perturbation analysis is available in Buonomo and LoSchiavo (2002) and Buonomo and LoSchiavo (2003). Furthermore, a PSpice simulation of the oscillator for $C=2 p F, L=4 n H, W_{n} / L_{n}=480$, $V_{T N}=0.5 \mathrm{~V}, V_{D D}=3 \mathrm{~V}$ and $k_{n}=\mu_{n} C_{o x}=20 \mu \mathrm{A} / \mathrm{V}^{2}$ is shown in Fig. 2. The initial point for the design is a detailed description of the components of the LC tank. Because of the structure of the circuit and under the assumption that the passive components are linear, the differential VCO can be reduced to the equivalent circuit shown in Fig. 3, see e.g. Hajimiri and Lee (1999). For the VCO presented in de Muer et al. (1998) follows

$$
\begin{aligned}
& g_{\text {tank }}=\frac{1}{2}\left(g_{\text {on }}+g_{\mathrm{v}, \text { max }}+g_{\mathrm{L}}\right) \\
& L_{\text {tank }}=2 L \\
& C_{\text {tank }}=\frac{1}{2}\left(C_{\mathrm{NMOS}}+C+C_{\mathrm{P}}+C_{V, \text { max }}+C_{\text {load }}\right)
\end{aligned}
$$

Published by Copernicus GmbH on behalf of the URSI Landesausschuss in der Bundesrepublik Deutschland e.V. 


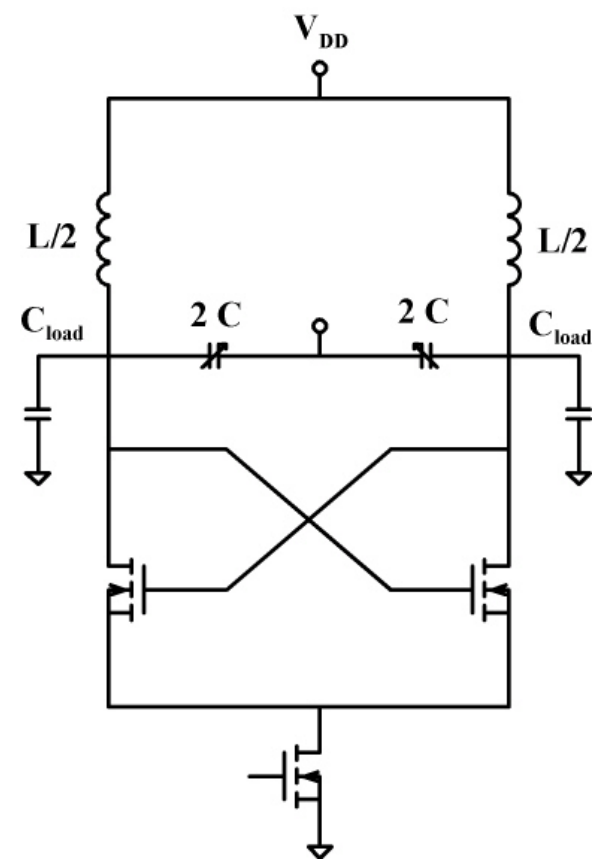

Fig. 1. CMOS cross-coupled LC tank oscillator.

where $C_{N M O S}=C_{g s, n}+C_{d b, n}+4 C_{g d, n}, \quad$ is the total parasitic capacitance, $g_{v}=C_{v, \max } \omega / Q_{v}=\omega^{2} C_{v}^{2} R_{v}$ and $g_{L}=1 / R_{p}+R_{S} /\left(\omega L_{s}\right)^{2}$ are the conductances of the varactors and inductors, respectively. If the transistors operate in the pinch-off region, the amplitude $V_{\text {tank }}$ is limited to $-V_{T N}<V_{\text {tank }}<V_{T N}$. As shown in Buonomo and LoSchiavo (2003) in that case the nonlinearity of the differential pair can be modeled by

$i(v)=-I_{0} \frac{v}{v_{\mathrm{n}}} \sqrt{\left(1-\frac{v^{2}}{v_{\mathrm{n}}^{2}}\right)}$

where $I_{0}$ is the bias current and $v_{n}=2\left(I_{0} L_{n} /\left(\mu_{n} C_{o x} W_{n}\right)\right)^{1 / 2}$. With Eq. (2) we are able to represent the VCO by a nonlinear dynamical system:

$$
\begin{aligned}
& \frac{d v}{d t}=-\frac{g_{\mathrm{tank}}}{C_{\mathrm{tank}}} v-\frac{1}{C_{\mathrm{tank}}} i+\frac{I_{0}}{C_{\mathrm{tank}}} \frac{v}{v_{n}} \sqrt{1-\frac{v^{2}}{v_{n}^{2}}} \\
& \frac{d i}{d t}=\frac{1}{L_{\mathrm{tank}}} v
\end{aligned}
$$

In order to apply our methodology first we have to approximate the nonlinearity by a series expansion, which can be represented by a cubic polynomial as shown in Buonomo and LoSchiavo (2002). Obviously the equilibrium point is given by $v^{*}=i^{*}=0$ and so we find

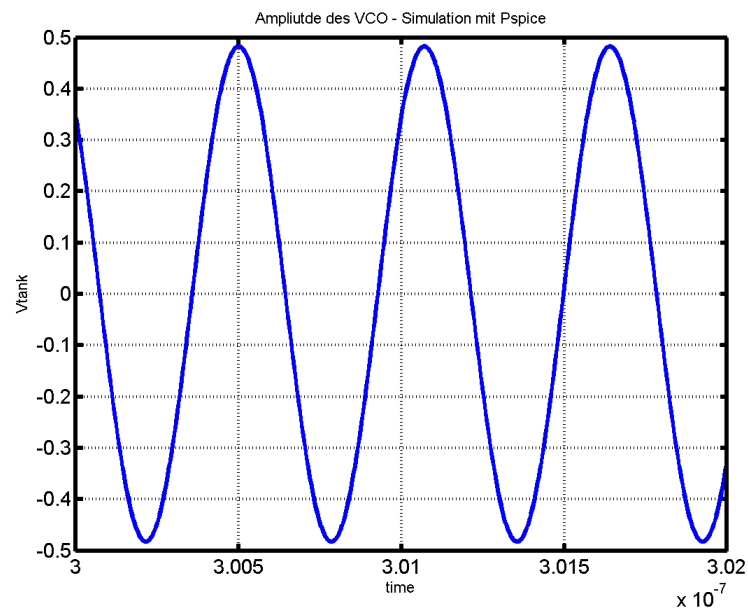

Fig. 2. Simulation of the steady state oscillation.

$$
\begin{aligned}
{\left[\begin{array}{c}
\frac{d v}{d t} \\
\frac{d i}{d t}
\end{array}\right]=} & {\left[\begin{array}{c}
-\frac{g_{\mathrm{tank}}}{C_{\mathrm{tank}}}+\frac{I_{0}}{C_{\mathrm{tank}} v_{n}}-\frac{1}{C_{\mathrm{tank}}} \\
\frac{1}{L_{\mathrm{tank}}} \\
0
\end{array}\right]\left[\begin{array}{l}
v \\
i
\end{array}\right] } \\
& +\left[\begin{array}{c}
\frac{1}{2} \frac{I_{0}}{C_{\mathrm{tank}}}\left(\frac{v}{v_{n}}\right)^{3} \\
0
\end{array}\right]+O\left(\left\|v^{5}\right\|\right)
\end{aligned} .
$$

\section{Bifuraction analysis}

The nonlinear analysis which is shown in the following is based on the Andronov Hopf theorem, see e.g. Mees and Chua (1979). Since the bifurcation analysis is similar to our investigation of VCOs in bipolar technology (Prochaska et al., 2005) here we outline only the basic results for CMOS LC tank oscillators. For a detailed description of our methodology the reader is left to Prochaska et al. (2005). It is a necessary condition of Hopf theorem that Eq. (4) possesses a pair of conjugate complex eigenvalues with vanishing real parts for a certain value of a suitable design parameter. Here, $\mu=-g_{\text {tank }} / C_{\text {tank }}+I_{0} /\left(C_{\text {tank }} v_{n}\right)$ is a useful choice. By means of the transformation $T=[0-1 ;-(L C) / L 0]$, $x=\left[\begin{array}{ll}v & i\end{array}\right]^{T}$ and $y=T x$ we find for $\mu=0$

$\dot{\mathbf{y}}=\left[\begin{array}{cc}0 & \omega_{0} \\ -\omega_{0} & 0\end{array}\right] \mathbf{y}+\left[\begin{array}{c}0 \\ k y_{2}^{3}\end{array}\right]$,

with $\omega_{0}=1 /-(L C)^{1 / 2}$ and $k=-1 / 2 / I_{0} /\left(C v_{n}^{3}\right)$. After that we have to test the stability of the equilibrium point. By means of the Poincare normal form, see e.g. Mathis (1995), we can calculate the Poincare coefficient $a_{1}=3 / 8 k$ which provides information about the stability of equilibrium point. If $a_{1}<0$ the equilibrium point is asymptotical stable and there is a variation of the bifurcation parameter $\mu$ which leads 
to a stable limit cycle. In order to predict the amplitude first by means of the transformation $T$ and for $\mu \neq 0$ we calculate

$\dot{\mathbf{y}}=\left[\begin{array}{cc}0 & \omega_{0} \\ -\omega_{0} & \mu\end{array}\right] \mathbf{y}+\varepsilon\left[\begin{array}{c}0 \\ k y_{2}^{3}\end{array}\right]$,

where we assume that a linear oscillator is perturbed by a small nonlinearity. Otherwise our approach for the prediction of the amplitude which is based on a perturbation method cannot be applied. For the further calculation it is necessary to mark perturbation by the perturbation parameter $\varepsilon$. Now we transform the given system into polar coordinates. For $y_{1}=r \sin \theta$ and $y_{2}=r \cos \theta$ it follows

$\dot{\theta}=\omega_{0}+\varepsilon\left(\begin{array}{c}\left(-\frac{1}{2} \mu-\frac{1}{4} k r^{2}\right) \sin 2 \theta \\ -\frac{1}{8} k \sin 4 \theta\end{array}\right)$
$\dot{r}=\varepsilon\left(\begin{array}{c}-\frac{1}{2} \mu r+\frac{3}{8} k r^{3}+\left(\frac{1}{2} \mu r+\frac{1}{2} k r^{3}\right) \\ \cos 2 \theta+\frac{1}{8} k r^{3} \cos 4 \theta\end{array}\right)$.

Since both equations of Eq. (7) are coupled, we cannot calculate the amplitude directly. However by means of averaging a relief can be produced as shown in Sanders and Verhulst (1985). For presentation higher order terms of the averaged system are neglected and so we arrive at

$\dot{\bar{\Theta}}=\omega_{0}$

$\dot{\bar{r}}=\varepsilon \bar{r} \mu-\varepsilon \frac{3}{8} k \bar{r}^{3}$

Since both equations of Eq. (8) are decoupled we are able to calculate a first approximation of the amplitude:

$$
\begin{aligned}
V_{\mathrm{tank}} \approx \bar{r} & =\sqrt{-\frac{4}{3} \frac{\mu}{k}} \\
& =4 \sqrt{\frac{2}{3} \frac{I_{0} L_{\mathrm{n}}}{k_{\mathrm{n}} W_{\mathrm{n}}}\left(1-\frac{2 g_{\mathrm{tank}}}{I_{0}} \sqrt{\frac{I_{0} L_{\mathrm{n}}}{k_{\mathrm{n}} W_{\mathrm{n}}}}\right)}
\end{aligned}
$$

If the stability condition given by the Poincare normal form is fulfilled Eq. (9) is valid. Otherwise the VCO possesses no limit cycle and the tank amplitude is zero. However, it must be pointed out that Eq. (9) is just a first approximation. By means of our approach the calculation of a sequence of averaging procedures is also possible which leads to a higher accuracy of the prediction of amplitude. Especially in that case our approach which is based on Lie series is advantageous, since the averaged system can be calculated in efficient manner, see e.g. Mathis and Voigt (1987). It must be also pointed out that the PSpice simulation and perturbation method performed in Buonomo and LoSchiavo (2002) validates our results. As given in Buonomo and LoSchiavo (2003) for the circuit shown in Fig. 1 follows $1 / g_{\text {tank }}=341 \Omega$. For example, by means of Eq. (9) we find $V_{\text {tank }}=0.4877 \mathrm{~V}$ which is a good approximation compared to the PSpice simulation shown Fig. 2.

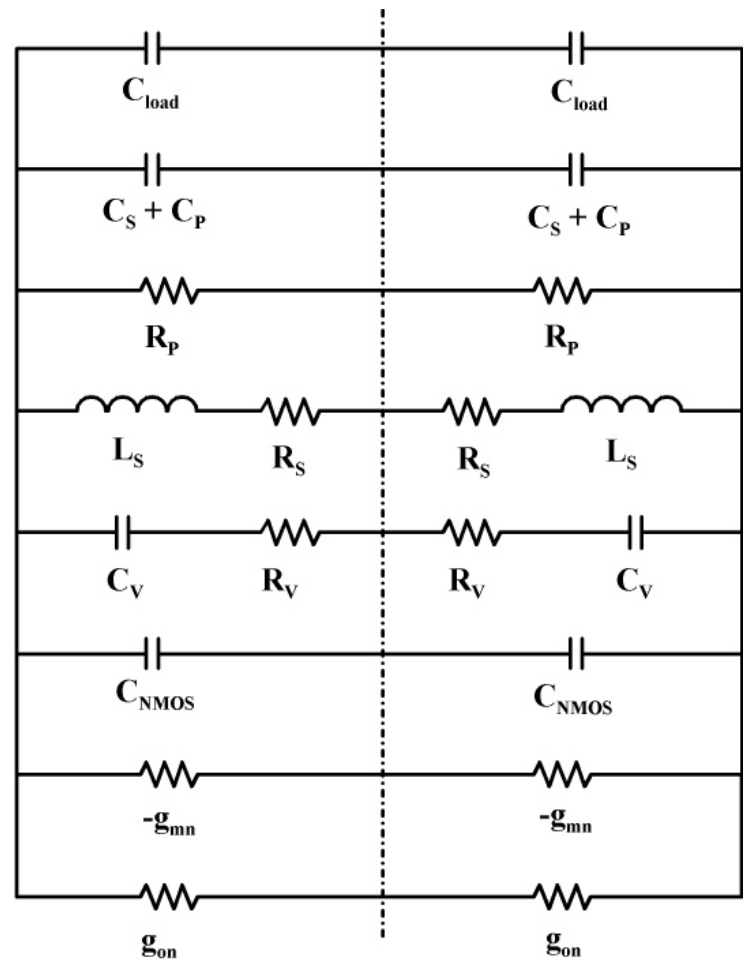

Fig. 3. Simplified equivalent circuit of LC tank VCOs.

\section{Design procedure}

As shown in Hajimiri and Lee (1999) and Ham and Hajimiri (2001) there are altogether 10 design variables: two for the MOS transistors $\left(W_{n}, L_{n}\right)$, the inductance $L_{\text {tank }}$ of spiral inductors given by geometric parameters $(b, s, n, d)$, the upper and lower limit of the varactors $\left(C_{v, \max }, C_{v, \min }\right)$, the load capacitors $C_{\text {load }}$ and the bias current $I_{0}$. These variables must be determined according to the specifications of the oscillator. Usually, it is reasonable to determine the design values with respect to the phase noise $L\left\{\omega_{\text {off }}\right\}$, where in our regime of operation the following equation holds, see Ham and Hajimiri (2001)]:

$L\left(\omega_{\text {off }}\right) \propto \frac{L_{\text {tank }}^{2} I_{0}}{V_{\text {tank }}^{2}}$

In order to analyze the dependency of the design variables on the phase noise in detail we put Eq. (9) into Eq. (10):

$L\left(\omega_{\text {off }}\right) \propto \frac{L_{\text {tank }}^{2}}{\frac{32}{3} \frac{L_{\mathrm{n}}}{k_{\mathrm{n}} W_{\mathrm{n}}}\left(1-2 g_{\operatorname{tank}} \sqrt{\frac{L_{\mathrm{n}}}{I_{0} k_{\mathrm{n}} W_{\mathrm{n}}}}\right)}$

From Eq. (11) follows that the phase noise can be minimized if

$\left(1-2 g_{\operatorname{tank}} \sqrt{\frac{L_{\mathrm{n}}}{I_{0} k_{\mathrm{n}} W_{\mathrm{n}}}}\right) \rightarrow \max$. 


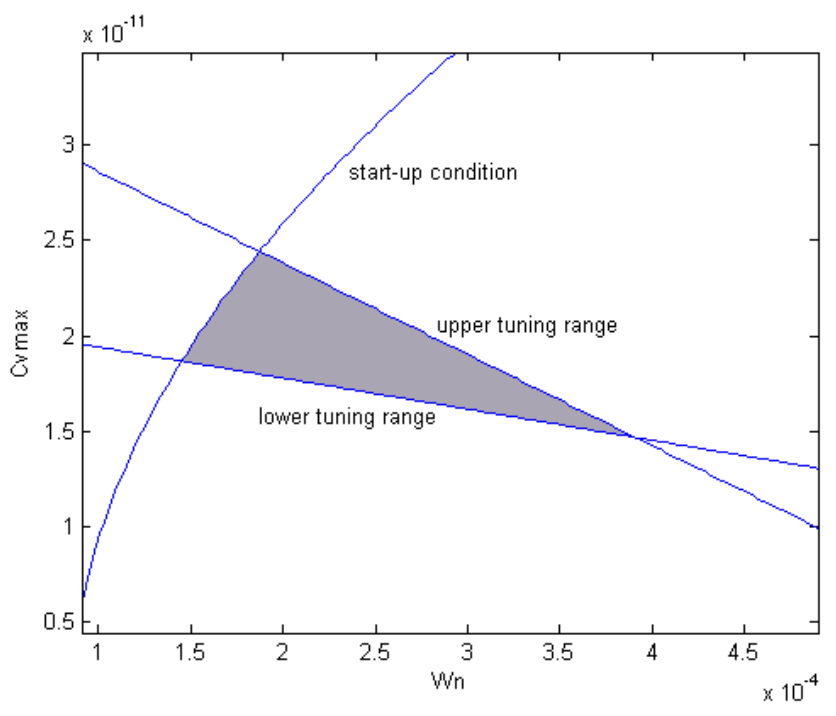

Fig. 4. CMOS cross-coupled LC tank oscillator.

It turns out that for given $g_{\operatorname{tank}}, W_{n}, L_{n}$ and $k_{n}$ Eq. (11) can be maximized if the bias current is set to its maximum value given by the specifications. From Eq. (12) also follows that the phase noise can be minimized by the choice of a very small bias current. However, in that case the amplitude tends to zero. To reduce parasitic capacitances and to get the highest transconductance in Hajimiri and Lee (1999) is suggested to set $L_{n}$ to the minimum value allowed by the process. However, as shown in Eq. (11) it must be taken into account that the phase noise also depends on $L_{n}$. After that the MOS transistors are described by only one independent design variable $W_{n}$. Since the ratio $C_{v, \text { max }} / C_{v \text {, min }}$ is typically determined by physical laws and remains constant for scalable layouts only one design variable $C_{v \text {, max }}$ is sufficient. Finally, the capacitance $C_{\text {load }}$ is directly given by the load and additionally assumptions as shown in Hajimiri and Lee (1999). Thus it remains the design variables $W_{n}, L_{\text {tank }}$ and $C_{v \text {,max }}$, where the inductance is given by the geometric parameters of the on-chip coils. After a suitable initial guess of the inductance value in Ham and Hajimiri (2001) three design steps are suggested in order to find the remaining design variables: First, the amplitude must be larger than a certain value $V_{\text {tank,min }}$ to provide a voltage swing adequate for the next stage $\left(V_{\text {tank }} \geq V_{\text {tank,min }}\right)$. Second, starting from the tuning range of the oscillation frequency one can define conditions for the upper and lower limit of tuning range

$L_{\mathrm{tank}} C_{\mathrm{tank}, \min } \leq \frac{1}{\omega_{\max }^{2}}, \quad L_{\mathrm{tank}} C_{\mathrm{tank}, \max } \geq \frac{1}{\omega_{\min }^{2}}$,

where the fractional tuning range is given by $\left(\omega_{\min }-\omega_{\max }\right) / \omega$ and $\omega=\left(\omega_{\max }-\omega_{\min }\right) / 2$. Third the startup condition

$g_{\text {active }} \geq \alpha_{\min } g_{\text {tank,max }}$

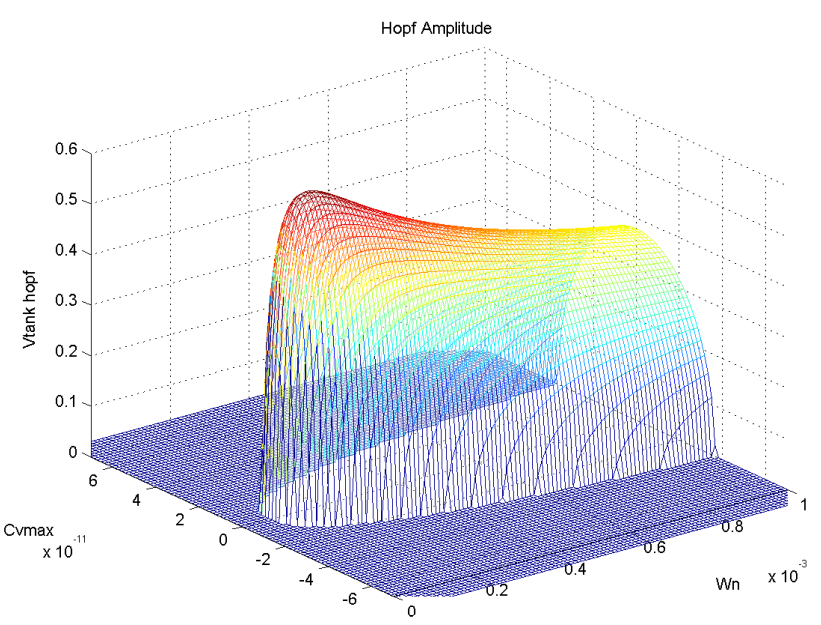

Fig. 5. Calculation of the tank amplitude in the design area, where higher harmonic are taken into account.

can also be used to get the remaining design variables. In Eq. (14) $\alpha_{\min }$ is the small-signal loop gain. The reader should note that Eq. (14) guarantees the start-up and the existence of steady state oscillation, where the latter is based on the Barkausen criterion as shown in Konstanznig et al. (2002). Since it can be shown that the Barkausen criterion is an integral part of the Hopf theorem our bifurcation analysis and the calculation of the amplitude is a more general formulation of Eq. (14). The difference between our technique and common methods are the consideration of nonlinear terms of higher degree. The visualization of the three design constrains in a $C_{v, \max }-W_{n}$ plane is shown in Fig. 4 , where the grey highlighted area - the so called feasible design area - marks all $\left(W_{n}, C_{v, \max }\right)$ which fulfill the design constrains. Since for the visualization of the start-up condition the choice of $L_{\mathrm{tank}}$ is necessary we have to pick an initial guess for the inductance value so that we find a suitable design area. In Ham and Hajimiri (2001) is recommended to choose a small inductance which maximize $g_{L}$ while in Craninchx and Steyaert (1995) a large inductance is preferred. For our modified design approach we also prefer an inductance selection as shown in Ham and Hajimiri (2001). However, by using our technique the initial choice of $L_{\text {tank }}$ is not important for the final result of the design process. So we suggest choosing the initial inductance in dependency on designer's experience of the application area to reduce the calculation effort during the design process. In general, the choice of $L_{\text {tank }}$ depends on the operating area of the oscillatory circuit. On the one hand it must be taken into account that with respect to Eq. (1) the VCO typically possesses for a smaller inductance a larger $g_{L}$. A minimized $g_{L}$ minimizes $g_{\text {tank }}$ and so we maximize Eq. (12). On the other hand Eq. (12) depends on the operating area given by $W_{n}$ and $C_{v \text {, max }}$. For example, if Eq. (12) is near its maximum the choice of a larger inductance leads 


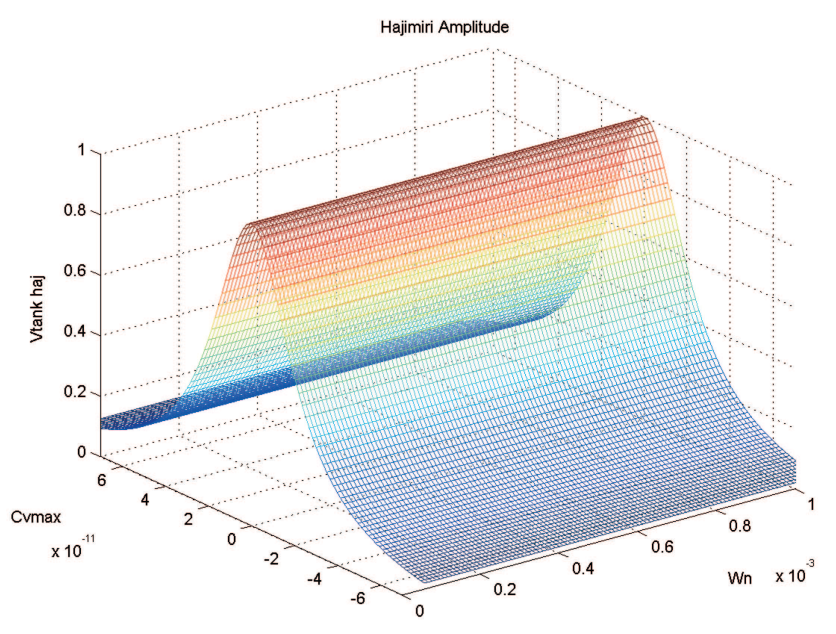

Fig. 6. Calculated Amplitude by describing function method.

to higher phase noise, since in that case a smaller conductance $g_{\text {tank }}$ does not increase Eq. (12) significantly. However, if Eq. (12) is small and $g_{\text {tank }}$ depends strongly on $g_{L}$ a larger $L_{\text {tank }}$ leads to a reduction of the phase noise since $g_{L}$ changes

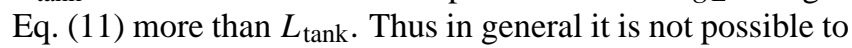
choose an ideal inductance value without knowledge about the other design variables. Unfortunately, $L_{\text {tank }}$ must be chosen in order to get the remaining design variables. A relief can be produced by using the results of our analysis, since phase noise depends on the amplitude significantly. Starting from Eq. (9) we are able to calculate the amplitude for every feasible $W_{n}-C_{v \text {, max }}$ pair of the design area (Fig. 5). Moreover, under consideration of robust design aspects we can calculate reliable $W_{n}-C_{v \text {, max }}$ pairs which lead to a maximum amplitude to guarantee best phase noise performance. After that we suggest to analyze Eqs. (11) and (12) with respect to Eq. (1) in order to decide if the inductance should be increased or decreased. By means of the modified $L_{\text {tank }}$ the design constrains can be adapted. This procedure must be repeated until the design area shrinks to single point or a further modification of $L_{\text {tank }}$ leads to no improvement. Especially in last case, the tank amplitude is an important criterion to choose a feasible $W_{n}-C_{v \text {, max }}$ pair. The reader should note that we are able to calculate the amplitude under consideration of higher harmonics which leads to a high accuracy of the design process. This is confirmed by the calculation of the amplitude by the well-known method of the describing function (Fig. 6), which leads to the big differences to the PSpice Simulation.

\section{Conclusions}

In this paper we have presented the first bifurcation analysis of fully integrated CMOS LC tank oscillators. Formulas for the calculation of the tank amplitude as well as the analysis of the stability are given which can directly be used for the design of differential VCOs. It turns out that the results of the analysis by an overall model of the active elements lead to an improvement of the design process, since by using our approach a deeper insight into the functionality of LC tank VCOs is provided. We have presented the results in an analytic form - designers are able to implement differential VCO by means of the given results even if other models of the components of the circuit are required. In this case possibly the calculations are more complicated. However, our methodology can be used in the same manner. So it represents a guide for the analysis and the design of electrical oscillators whereas the nonlinearities of circuit components are an integral part of the design process. It turns also out that geometric methods are powerful tools for investigations of nonlinear oscillators. Since they provide a survey of the solution set we get a deeper insight in the behavior of the network. In contrast to numerical computation the shown analytical methods have the advantage that the results are interpretable by the parameters of the network. Furthermore, we have implemented geometric methods by means of computer algebra. Because of these routines the calculations proceed in an automated manner.

\section{References}

Buonomo, A. and Lo Schiavo, A.: Analyzing the Dynamic Behavior of RF Oscillators, IEEE Trans. on Cir. and Sys., 49, 15251534, 2002.

Buonomo, A. and Lo Schiavo, A.: Determining the oscillation of differential VCOs, Proc. IEEE Intern. Symp. on Cir. and Sys. (ISCAS 2003), 3, 25-28, 2003.

Craninckx, J. and Steyaert, M.: Low-Noise Voltage-Controlled Oscillators Using Enhanced LC-Tanks, IEEE Trans. on Cir. and Sys. - Analog and Digital Signal Processing, 42, 794-804, 1995.

Hajimiri, A. and Lee, T. H.: The design of low noise oscillators, Kluver Academic Publishers, Boston, 1999.

Ham, D. and Hajimiri, A.: Concepts and Methods in Optimization of Integrated LC VCOs, IEEE J. of Solid-State Circuits, 36, 896908, 2001.

Konstanznig, G., Pappenreiter, T., Maurer, L., Springer, A., and Weigel, R.: Design of a $1.5 \mathrm{~V}, 1.1 \mathrm{~mA}$ Fully Integrated LCtuned Voltage Controlled Oscillator in a $4 \mathrm{GHz}-$ Band using a 0.12 m CMOS-Process, Asian-Pacific Microwave Conf., 14711474, 2002.

Mathis, W.: Transformation and Equivalence, in: The Circuits and Filter Handbook, edited by Chen, W. K., CRC Press, Boca Raton, 1995.

Mathis, W. and Russer, P.: Oscillator Design, in: Encyclopedia of RF and Mircowave Engeenering, edited by Chang, K., John Wiley, New York, 4, 3563-3589, 2005.

Mathis, W. and Voigt, I.: Applications of Lie Series Averaging in Nonlinear Oscillations, Proc. IEEE Intern. Symp. on Cir. and Sys. (ISCAS 1987), Philadelphia, 1987. 
Mees, A. I. and Chua, L. O.: The Hopf Bifurcation and its Application to nonlinear Oscillations, IEEE Trans. on Cir. and Sys., 26, 235-254, 1979.

de Muer, B., Borremanns, M., Steyaert, M., and Puma, G. Li: A $1.8 \mathrm{GHz}$ highly-tunable low-phase-noise CMOS VCO, IEEE Customs Integrated Circuits Conf., 585-588, 1998.

Prochaska, M., Belski, A., and Mathis, W.: Bifurcation Analysis of On-Chip LC VCOs, IEEE Intern. Symp. on Circuits and Systems (ISCAS 2005), May 23.-26., Kobe, Japan, 2005.
Razavi, B.: RF Microelectronics, Prentice-Hall, Englewood Ciffs, New York, 1997.

Sanders, J. A. and Verhulst, F.: Averaging Methods in Nonlinear Dynamical Systems, Springer Verlag, New York, 1985.

van der Tang, J., Kasperkovitz, D., and van Roermund, A.: HighFrequency Oscillator Design for Integrated Transceivers, Kluwer Academic Publishers, Boston, 2003.

Tietze, U. and Schenk, C.: Electronic Circuits: Handbook for Design and Application, Springer Verlag, New York, 2004. 